\title{
Indocyanine green videoangiography for recipient vessel stratification in superficial temporal artery-middle cerebral artery bypass surgery
}

\author{
Johannes Goldberg, MD, ${ }^{1,2}$ Peter Vajkoczy, MD, ${ }^{1}$ and Nils Hecht, MD'1 \\ 1Department of Neurosurgery and Center for Stroke Research Berlin (CSB), Charité Universitätsmedizin Berlin, Germany; and \\ 2Department of Neurosurgery, Inselspital, Bern University Hospital, University of Bern, Switzerland
}

\begin{abstract}
OBJECTIVE In superficial temporal artery-middle cerebral artery (STA-MCA) bypass surgery, recipient vessel properties are likely one of the main reasons for bypass failure. In daily practice, most surgeons select the recipient with the largest diameter. However, selection of the ideal recipient remains debatable because there are no objective selection criteria if multiple potential recipients exist. Here, the authors assessed the benefit of using indocyanine green videoangiography (ICG-VA) to optimize recipient vessel selection in patients undergoing STA-MCA bypass surgery for hemodynamic compromise.
\end{abstract}

METHODS All patients who had undergone STA-MCA bypass procedures with pre- and postanastomosis ICG-VA between 2010 and 2019 were eligible for inclusion in this study. The primary bypass surgeon was blinded to the preanastomosis ICG-VA. Preanastomosis white-light and ICG-VA images were compared to determine the identifiability of potential recipient vessels and pathological flow patterns. After completion of the anastomosis, a second (postanastomosis) ICG-VA image was used to analyze the flow increase within the chosen recipient based on the vessel diameter, initial recipient blood flow, initial sequence of appearance on ICG-VA, initial blood flow direction within the recipient, and orientation of the bypass graft. ICG-VA, FLOW 800, and intraoperative white-light images, as well as demographic, clinical, and radiographic patient data, were retrospectively analyzed by a clinician who was not directly involved in the patients' care.

RESULTS Sixty patients underwent 65 STA-MCA bypass procedures with pre- and postanastomosis ICG-VA. The ICGVA permitted identification of a significantly higher number of potential recipient vessels (median 4, range 1-9) than the white-light images (median 2, range 1-5; $p<0.001$ ), with detection of pathological flow patterns in $20 \%$ of all procedures. No association was found between the diameter and blood flow within potential recipients (Spearman $r=0.07, p=0.69$ ). After bypass grafting, the highest flow increase was noted in recipients with an initially low flow $(p<0.01)$, a late appearance $(p<0.01)$, and an initially retrograde flow direction $(p=0.02)$. Interestingly, flow increase was not significantly influenced by the recipient diameter $(p=0.09)$ or graft orientation $(p=0.44)$.

CONCLUSIONS ICG-VA facilitates identification of potential recipient vessels and detection of pathological flow patterns. Recipients with an initially low flow, a late appearance, and a retrograde flow seem to bear the highest potential for flow increase, possibly due to a higher hemodynamic need for revascularization.

https://thejns.org/doi/abs/10.3171/2020.5.JNS20642

KEYWORDS indocyanine green videoangiography; STA-MCA bypass; recipient vessel; chronic cerebral ischemia; hemodynamic stroke; cerebral revascularization; vascular disorders

$\mathrm{A}$ s suggested by Charbel and colleagues, poor bypass function likely results from one of two types of error: 1) poor indication for revascularization or 2) technical problems with the donor, the anastomosis, and/or the recipient. ${ }^{1}$ Although functional cerebral blood flow diagnostics, ${ }^{2}$ flow assessment with ultrasound, ${ }^{1,3}$ and microsurgical training ${ }^{4}$ have helped to limit these errors, there are still no objective criteria to guide selection of the ideal recipient.

Recipient selection generally requires two steps: first, reliable identification of all potential recipients within the exposed surgical field, and second, selection of a suitable recipient. In daily practice, most surgeons choose the recipient based on personal experience and primarily under

ABBREVIATIONS ACVD = arteriosclerotic cerebrovascular disease; $\mathrm{Al}=$ arbitrary intensity; ICG-VA = indocyanine green videoangiography; MMV = moyamoya vasculopathy; $\mathrm{ROI}$ = region of interest; STA-MCA = superficial temporal artery-middle cerebral artery.

SUBMITTED February 29, 2020. ACCEPTED May 18, 2020.

INCLUDE WHEN CITING Published online August 28, 2020; DOI: 10.3171/2020.5.JNS20642. 
consideration of diameter, location of the recipient within the surgical field, and morphological appearance of the vessel. Here, the use of image guidance and augmented reality has recently been shown to be useful for preoperative surgical planning and recipient selection focusing on a large vessel diameter and a targeted exposure. ${ }^{5-7}$ However, image guidance and augmented reality cannot determine flow-specific characteristics within potential recipients, which could provide additional useful criteria to optimize recipient selection in patients with hemodynamic need. For this purpose, indocyanine green videoangiography $(\mathrm{ICG}-\mathrm{VA})^{8}$ with FLOW $800^{9}$ may serve as a simple, safe, and cost-effective technique for immediate visualization and semiquantitative mapping of macro-circulatory flow beyond white-light visualization alone..$^{10-15}$ In the present study, we used ICG-VA and FLOW 800 for pre- and postanastomosis recipient vessel characterization to identify specific recipient vessel properties that could be beneficial for superficial temporal artery-middle cerebral artery (STA-MCA) bypass function in patients with hemodynamic compromise.

\section{Methods}

\section{Study Design and Patient Population}

This study was approved by the local ethics committee of the Charité University Hospital in Berlin, Germany, and performed in compliance with Health Insurance Portability and Accountability Act regulations. We included in this study all patients who had undergone an STA-MCA bypass procedure for flow augmentation of cerebral hemodynamic impairment between 2010 and 2019 and in whom pre- and postanastomosis ICG-VA images were available. In general, criteria for revascularization were 1) moyamoya vasculopathy (MMV) or stenoocclusive arteriosclerotic cerebrovascular disease (ACVD), 2) recurrent transient ischemic attacks under the best medical management, and 3) impaired cerebrovascular reactivity confirmed by PET or SPECT during acetazolamide challenge. Bypass patency at follow-up (12-18 months) was categorized according to the degree of MCA territory filling. ${ }^{16}$ ICG-VA, FLOW 800, and intraoperative white-light images, as well as demographic, clinical, and radiographic patient data, were retrospectively analyzed by a clinician who was not directly involved in the patients' care.

\section{STA-MCA Bypass Grafting}

Standardized end-to-side STA-MCA bypass grafting was performed as previously described..$^{17,18}$ After dissection, mobilization, and temporary clipping of the frontal or parietal branch of the STA, ${ }^{19}$ exposure of the recipient was gained through a 3- to 4-cm craniotomy centered above the distal end of the sylvian fissure according to the cortical target point, ${ }^{20}$ and a first (preanastomosis) ICG-VA was performed. Preanastomosis ICG-VA was performed after opening the dura mater at the discretion of the surgeon, who performed the procedure up to the point when the primary bypass surgeon joined to select the recipient vessel based on white light and complete the anastomosis. Importantly, the bypass surgeon was blinded to the preanastomosis ICG-VA and selected the recipient under white light based on personal experience, in terms of recipient accessibility and diameter. The selected recipient was prepared for the anastomosis by sharp arachnoid dissection. Small cortical branches originating within the designated anastomosis site were coagulated and cut to allow complete circumferential mobilization and placement of a blue, rubber flap below the designated anastomosis site. Next, the temporary clip across the distal end of the donor was briefly removed to confirm antegrade flow and then repositioned across the proximal donor vessel to permit fish-mouth incision and flushing of the donor with papaverine hydrochloride. Next, the inspired fraction of oxygen was adjusted to $100 \%\left(\mathrm{FiO}_{2} 1.0\right)$ for a 1- to 3-minute preoxygenation period. Then, Sugita AVM clips (Mizuho Medical Co. Ltd.) were placed across the proximal and distal recipient anastomosis site, and a longitudinal arteriotomy was performed with microscissors after gently lifting the lateral vessel wall with a 10-0 nylon suture (Ethilon, Ethicon Inc.). For grafting of the end-toside anastomosis under intermittent papaverine hydrochloride irrigation, 4 "outside-in-inside-out" pilot sutures were placed from the heel, toe, frontside, and backside of the donor to the recipient. Importantly, the orientation of the fish-mouthed donor was chosen based on anatomical considerations alone, such as orientation of the recipient or length of the prepared donor vessel. The anastomosis was then completed using interrupted or running 10-0 nylon sutures. The distal and proximal temporary clips across the recipient were removed after completion of the anastomosis, and the patency of the anastomosis was visually assessed based on recipient filling and backflow into the donor. Thereafter, bypass flow was initiated by removing the temporary clip across the donor, and bypass patency was assessed by performing a second (postanastomosis) ICG-VA (Fig. 1).

\section{Acquisition of ICG-VA and Analysis of FLOW 800-Specific Hemodynamic Parameters}

Pre- and postanastomosis ICG-VA was performed with a commercially available operating microscope (OPMI Pentero 900, Carl Zeiss) equipped with a fluorescent light source (wavelength 700-850 $\mathrm{nm}$ ) and an infrared-sensitive camera. The microscope was positioned perpendicular to the area of investigation at a distance of approximately $300 \mathrm{~mm}$. With each ICG-VA, a corresponding white-light image was acquired. During ICG-VA, room lights were dimmed and a body weight-adapted dose of $0.25 \mathrm{mg} / \mathrm{kg}$ ICG (Pulsion Medical Systems) dissolved in 5 $\mathrm{ml}$ of distilled water was injected through a central venous catheter as a bolus, followed by a $10-\mathrm{ml}$ bolus of sterile saline. The color-coded FLOW 800 blood flow map was automatically generated but was analyzed postoperatively: for the purpose of determining FLOW 800-specific hemodynamic parameters, predefined square regions of interest (ROIs) were manually placed within the potential and the definite recipient before and after bypass grafting, respectively (Fig. 2). Next, semiquantitative recipient flow was calculated according to the method of Kuebler and colleagues: ${ }^{21}$ maximum fluorescence intensity (AI) $\div$ rise time (seconds) $=$ semiquantitative flow $(\mathrm{AI} / \mathrm{sec})$, where AI refers to arbitrary intensity. Rise time was defined as 

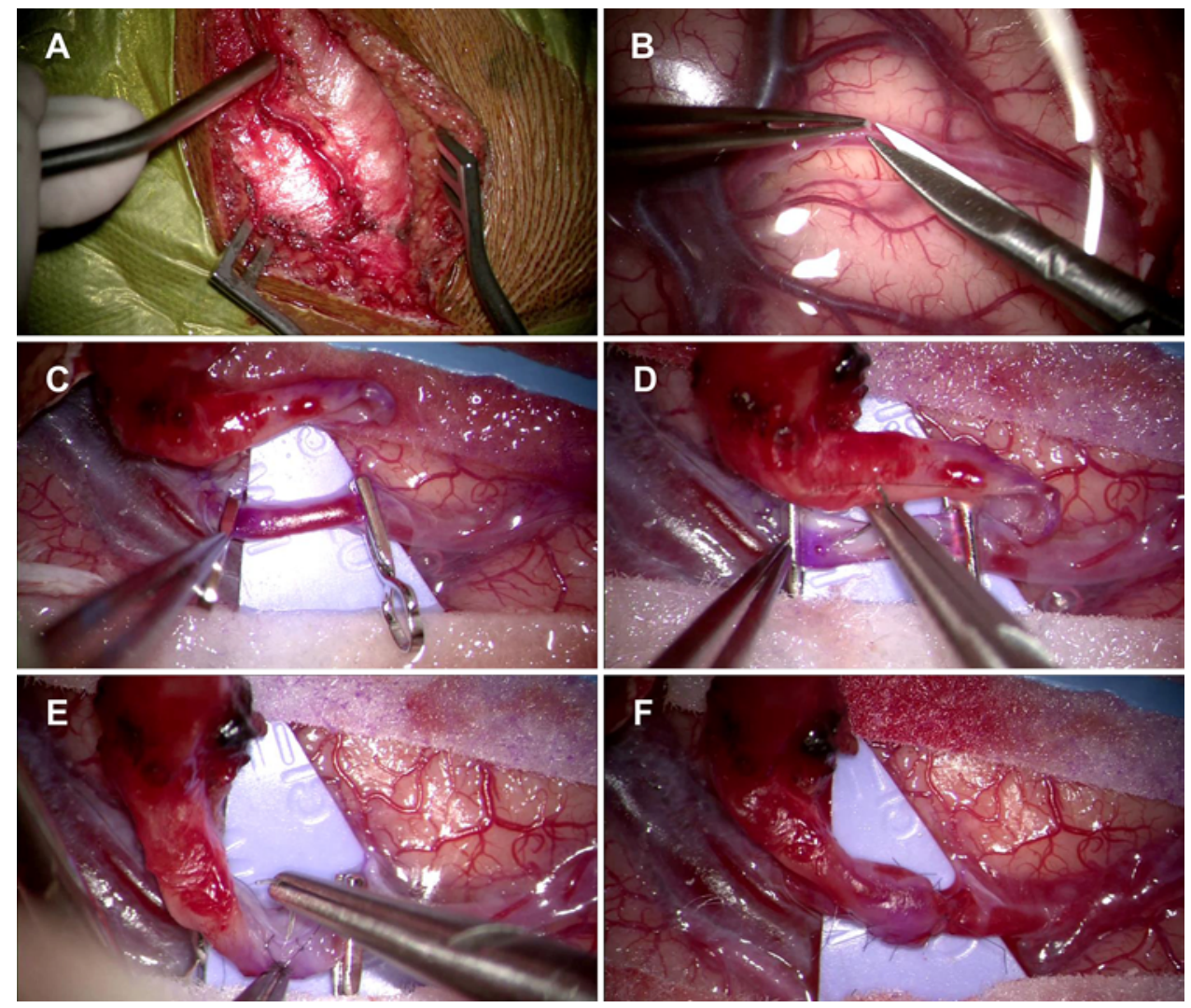

FIG. 1. Step-by-step illustration of the surgical technique for STA-MCA bypass grafting: preparation of the donor (A), sharp arachnoid dissection for preparation of the recipient (B), temporary clipping (C), "outside-in-inside-out" pilot suture at the heel with 10-0 nylon (D), suturing of the anastomosis (back wall) with interrupted 10-0 nylon (E), and completed anastomosis with temporary clips removed $(\mathbf{F})$. Figure is available in color online only.

the time interval between $10 \%$ and $90 \%$ of the maximum fluorescence intensity. ${ }^{12}$ We deliberately measured semiquantitative recipient flow in AI per second because AI is based on the absolute value of the fluorescence intensity and remains less prone to interindividual variability caused by differences in cardiac output or bolus injection.

\section{Analysis of White-Light Images, ICG-VA, and FLOW 800}

Preanastomosis white-light images and ICG-VA were compared in terms of the overall identifiability of potential recipients and pathological flow patterns. Pathological flow patterns were assessed with ICG-VA and defined as any nonlinear flow of the fluorescent dye or irregular configuration of the vessel wall leading to the three main categories-thrombosis, spasm, and stagnant flow-as well as clearly evident pathological flow patterns that could not be assigned to one of these groups. Vessels within the surgical field were visually analyzed by an experienced bypass surgeon. Categorization as a potential recipient was generally made considering properties necessary to perform microanastomosis, such as diameter, location in the surgical field, and morphological appearance. The blinded primary bypass surgeon of this study was not involved in the reviewing process. To facilitate an interindividual comparison, the following parameters for recipient selection were broken down into 3 ranks: semiquantitative flow
(AI/sec) within the recipient (i.e., flow rank), sequence of appearance (i.e., sequence rank), and vessel diameter according to preanastomosis ICG-VA (i.e., diameter rank).

Flow ranks were obtained by measuring flow in all potential recipient vessels in each surgical field by using FLOW 800. Potential recipients with the highest flow in each field were assigned the rank of 1; recipients with the second highest flow, the rank of 2, and so on. Sequence and diameter ranks were obtained by visual analysis of ICG-VA. Accordingly, recipient vessels with the earliest appearance on ICG-VA (sequence rank) or largest diameter (diameter rank) were assigned the highest rank of 1 . Recipients that appeared second on ICG-VA or had the second largest diameter were assigned the rank of 2 , and so on.

The effect of specific recipient properties on bypass function was determined by comparing flow within the definite recipient before and after completion of the anastomosis: to permit an interindividual comparison of FLOW 800-specific hemodynamic parameters, baseline semiquantitative flow $(\mathrm{AI} / \mathrm{sec})$ was first normalized to "relative recipient flow" (\%), which we defined as the percentage of flow within the definite recipient in relation to the highest semiquantitative flow among all potential recipients in the same patient. Next, the "recipient flow increase" $(\Delta \%)$ was calculated as the percent flow increase 


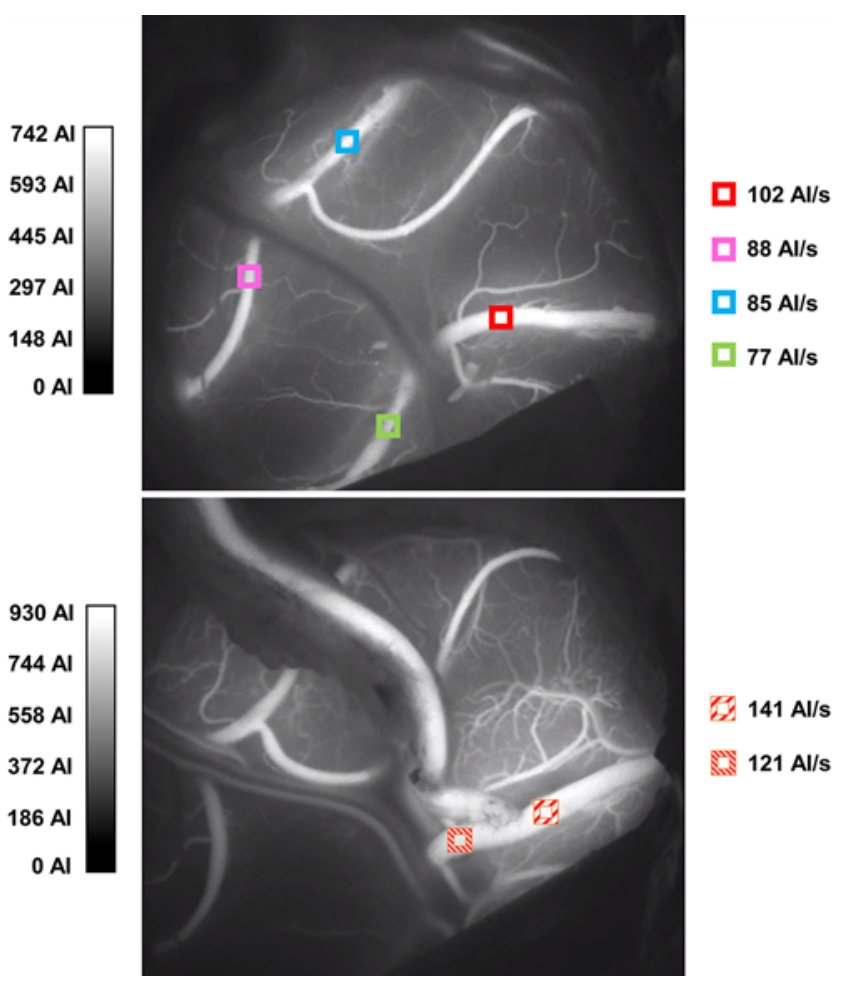

FIG. 2. Illustration of hemodynamic recipient blood flow analysis based on preanastomosis (upper) and postanastomosis (lower) ICG-VA. The left legend indicates the range of arbitrary intensity (AI) on a scale from black to white for each ICG-VA. The right legend displays flow measurements in $\mathrm{Al}$ per second (Al/s) in each individually placed $\mathrm{ROI}$ within the ICG-VA. In the upper panel, ROIs are placed within potential recipients. In the lower panel, ROIs are placed into the chosen recipient, both proximal and distal to the anastomosis site. Figure is available in color online only.

within the recipient after completion of the anastomosis. Then, the association between the recipient ranks and the degree of recipient flow increase was determined by plotting the flow, sequence, and diameter ranks against the recipient flow increase.

To investigate the effect of blood flow direction and bypass graft orientation on recipient flow increase, the preanastomosis blood flow direction in the chosen recipient was categorized as antegrade or retrograde in relation to the sylvian fissure, and the orientation of the bypass graft was categorized as complementary or opposing depending on the orientation of the heel and toe of the donor in relation to the preanastomosis blood flow direction within the recipient.

\section{Statistical Analysis}

Descriptive summary statistics are presented as mean \pm standard deviation, median and range (minimum-maximum), percentage, or frequency, as appropriate. For comparison between recipient count in white-light and ICGVA images, a two-tailed Wilcoxon signed-rank test was used. To describe the association between diameter rank and semiquantitative flow, Spearman's correlation analysis was performed. To analyze the recipient flow increase after completion of the anastomosis, a paired t-test was
TABLE 1. Baseline and follow-up characteristics among 60 patients who underwent STA-MCA bypass

\begin{tabular}{lc}
\hline \multicolumn{1}{c}{ Characteristic } & Value \\
\hline Female, no. of patients (\%) & $32(53)$ \\
\hline Male, no. of patients (\%) & $28(47)$ \\
\hline Mean age in yrs (SD) & $39( \pm 18)$ \\
\hline Age range in yrs & $1-67$ \\
\hline Indication for surgery, no. of procedures (\%) & $65(100)$ \\
\hline MMV & $44(68)$ \\
\hline ACVD & $21(32)$ \\
\hline Angiographic FU rating at 12-18 mos, no. of procedures & $61(100)$ \\
(\%) & $2(3)$ \\
\hline 0, occluded & $5(8)$ \\
\hline 1, 1 cortical branch & $28(46)$ \\
\hline 2, ante- \& retrograde filling of MCA territory & $26(43)$ \\
\hline 3, filling of entire MCA territory & $59(97)$ \\
\hline Bypass patency, no. of procedures (\%) & 103 \\
\hline Cumulative FU in yrs
\end{tabular}

$\mathrm{FU}=$ follow-up

used. For comparisons of flow, sequence, and diameter ranks depending on recipient flow increase, a one-way ANOVA with Holm-Šidák's multiple comparison test was used. For comparison of blood flow direction and bypass orientation depending on recipient flow increase, an unpaired t-test was used. All tests were two-tailed. Statistical analyses were performed using GraphPad Prism version 8.0.1 and IBM SPSS Statistics version 25 (IBM Corp.). Statistical significance was set at $\mathrm{p}<0.05$.

\section{Results}

\section{Patient Characteristics}

Between 2010 and 2019, 60 consecutive patients underwent 65 STA-MCA bypass procedures with pre- and postanastomosis ICG-VA available. Thirty-two patients (53\%) were female. Forty-four procedures $(68 \%)$ were performed for the treatment of MMV and 21 (32\%) for ACVD. The mean patient age was $39 \pm 18$ years (range 1-67 years). Ten patients $(17 \%)$ belonged to the pediatric age group ( $<18$ years). Late follow-up at $12-18$ months was available for 61 procedures in 56 patients (93\%) and yielded a bypass patency rate of $97 \%$ (Table 1). The perioperative stroke rate at discharge and late follow-up was $6.2 \%$ (4 of 65 cases) with a risk of disabling stroke at $3.1 \%$.

\section{ICG-VA for Recipient Selection}

ICG-VA allowed identification of a significantly higher number of potential recipients (median 4, range 1-9) than white-light visualization alone (median 2, range 1-5; $\mathrm{p}<$ 0.001 ). Pathological flow patterns within a potential recipient were identified in 13 of 65 procedures (20\%) and included 3 potential recipients with intraluminal thrombus (23\%), 5 with stagnant or sluggish flow (38\%), 3 with spasm (23\%), and 2 with evident pathological flow that could not be further distinguished (15\%). In $>45 \%$ of all procedures, the primary bypass surgeon chose the recipi- 


\section{Flow rank (ICG/Flow 800) Sequence rank (ICG) Diameter rank (ICG)}
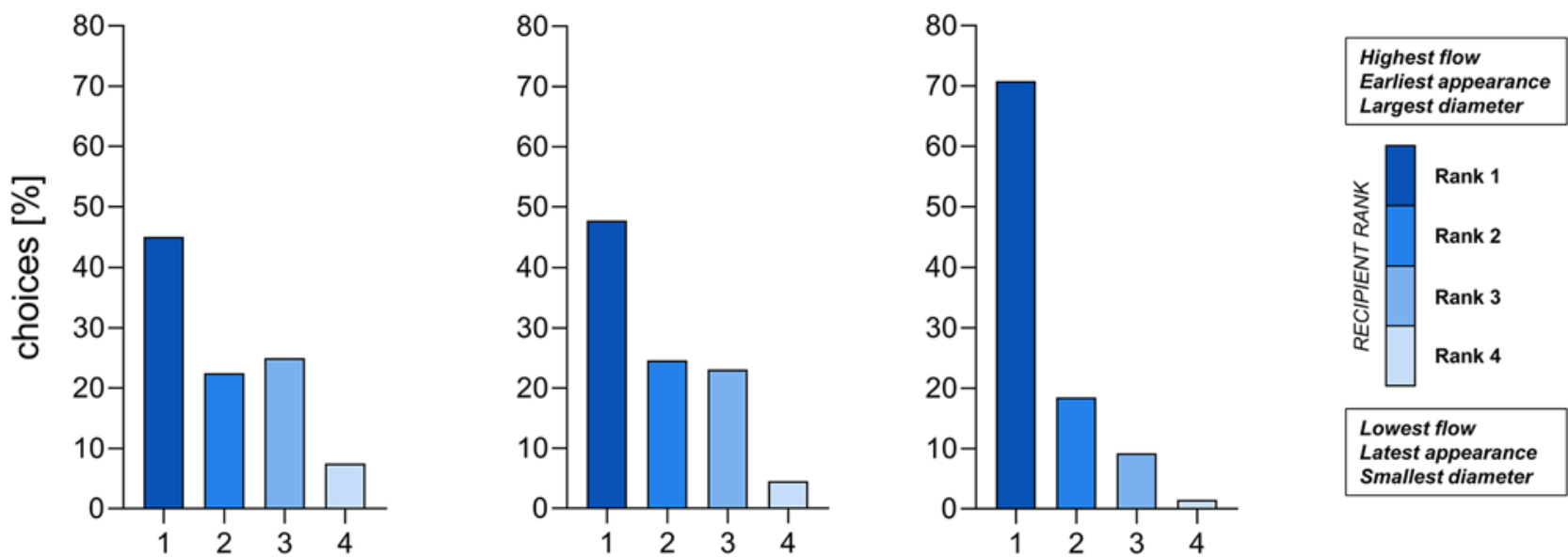

FIG. 3. Bar graphs illustrating the primary bypass surgeons' recipient selection depending on flow, sequence, and diameter ranks. The $y$-axis shows the surgeons' selection percentage. The x-axis represents flow, sequence, or diameter ranks stratified by ICGVA. Figure is available in color online only.

ent with the highest flow, earliest ICG-VA appearance, and largest diameter on ICG-VA (Fig. 3). The largest diameter represented the primary surgeons' single most frequently applied criterion $(71 \%)$ for recipient selection based on white light. Interestingly, no association was found between the diameter and preanastomosis flow within the potential recipients (Spearman $\mathrm{r}=0.07, \mathrm{p}=0.69$ ).

\section{Influence of Recipient Vessel Characteristics on Bypass Function}

First, we analyzed baseline flow (relative recipient flow) within potential recipients and the flow increase within the chosen recipient. The mean range of relative recipient flow among potential recipients in the same procedure was $26 \% \pm 16 \%$ (range $0 \%-68 \%$ ), and bypass grafting resulted in a significant recipient flow increase of $70 \% \pm$ $100 \%$ ( $p<0.001$ for pre- vs postanastomosis relative recipient flow; Fig. 4). The primary diagnosis had no influence on bypass function (recipient flow increase; $p=0.2$ for MMV vs ACVD).
Second, we investigated the effect of vessel diameter, initial recipient blood flow, and initial sequence of appearance on ICG-VA on bypass function (recipient flow increase), which revealed that recipients with the initially lowest preanastomosis flow (rank 4) and latest ICG-VA appearance (rank 3) demonstrated a significantly greater flow increase than recipients with the highest flow (rank 1 [ $p<0.01]$ vs rank 4) and earliest appearance (rank 1 [p $<0.01]$ vs rank 3 ). The diameter of selected recipients had no influence on the postanastomosis flow increase ( $\mathrm{p}$ $=0.09$; Fig. 5).

Third, we analyzed the effect of the initial recipient blood flow direction and the orientation of the bypass graft on bypass function (recipient flow increase). The preanastomosis blood flow direction in chosen recipients was antegrade in 54 of 65 procedures $(83 \%)$ and retrograde in 11 of 65 procedures (17\%). Complementary bypass orientation was noted in 40 of $65(61 \%)$ and opposing orientation in 25 of 65 procedures (39\%). After revascularization, a significantly higher recipient flow increase was observed
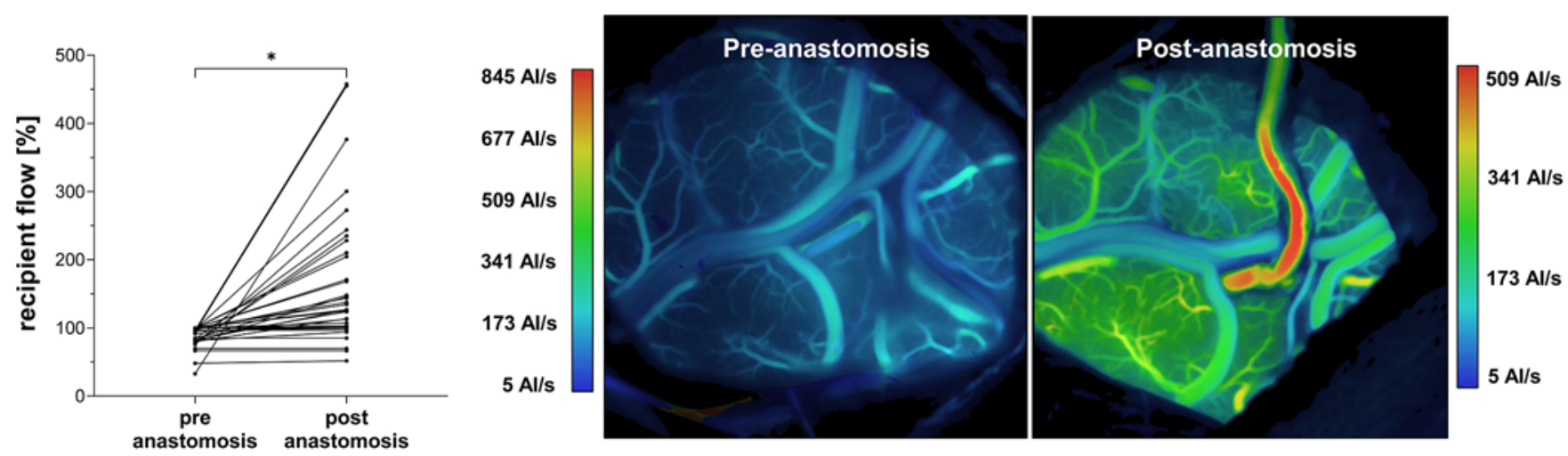

FIG. 4. Left: The significant but heterogeneous distribution of the recipient flow increase based on the pre- and postanastomosis relative recipient flow ( $p$ < 0.001). Right: A color-coded FLOW 800 blood flow map of a pre- and postanastomosis ICG-VA to visualize the flow increase before and after bypass grafting. The legends indicate the individual range of Al units on a color-coded scale for each ICG-VA. Figure is available in color online only. 
Flow rank (ICG/Flow 800) Sequence rank (ICG) Diameter rank (ICG)
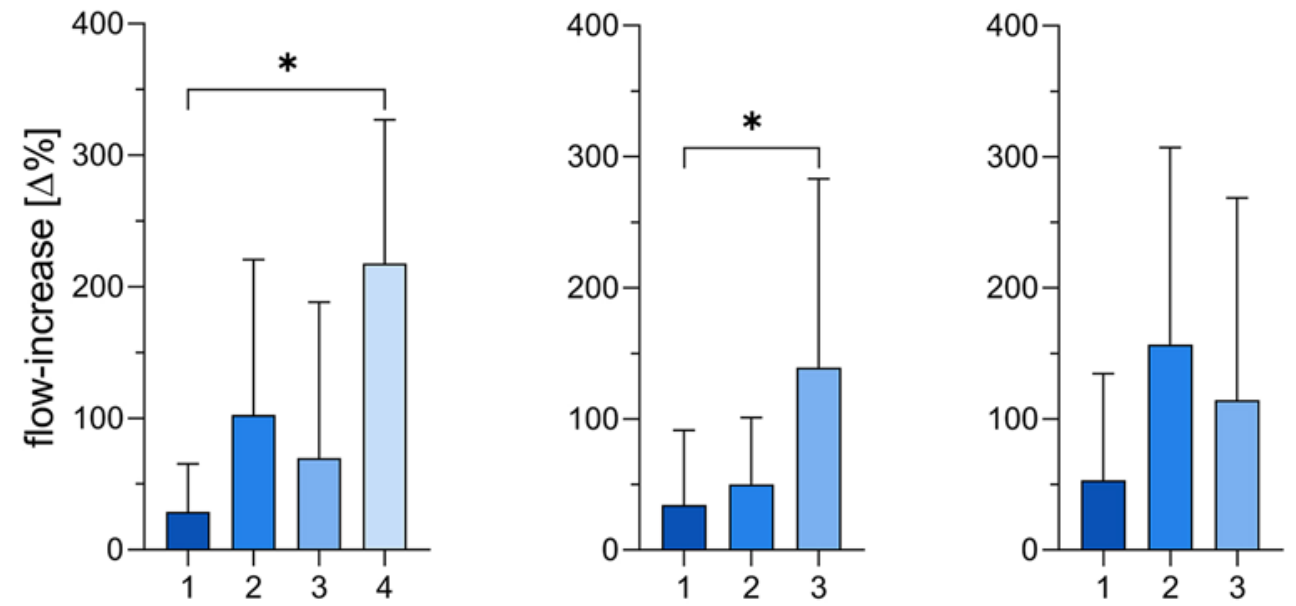

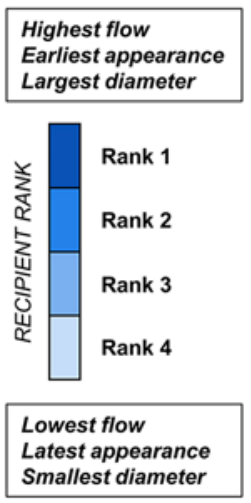

FIG. 5. Bar graphs illustrating the recipient flow increase depending on flow, sequence, and diameter ranks ( $\left.{ }^{*} p<0.01\right)$. The $y$-axis represents the recipient flow increase in percent. The $x$-axis represents flow, sequence, and diameter ranks stratified by ICG-VA. Figure is available in color online only.

in recipients with an initially retrograde blood flow direction (flow reversal toward the sylvian fissure; $p=0.02$ for antegrade vs retrograde), but no difference was noted depending on the graft orientation ( $\mathrm{p}=0.44$ for complementary vs opposing; Fig. 6).

\section{Discussion}

In this study, we show the usefulness of ICG-VA for improved identification of recipient vessels and pathological recipient flow patterns during STA-MCA bypass surgery in patients with hemodynamic compromise. Importantly, semiquantitative blood flow analysis of potential and selected recipients revealed that recipients with an initially low flow, late appearance on ICG-VA, and retrograde blood flow direction had the greatest recipient flow increase, which, unexpectedly, was not influenced by recipient diameter or bypass graft orientation.

Selection of an ideal recipient is likely one of the main determinants of bypass function. ${ }^{13,13}$ Despite multiple applications to study bypass patency, hemodynamic changes, and recipient identification in revascularization surgery, ${ }^{11-14,22-25}$ this is the first study that uses ICG-VA to derive objective recipient selection criteria for STAMCA revascularization in patients with hemodynamic compromise. Importantly, ICG-VA permitted improved identification of recipients, particularly in cases in which identifiability under white light was impaired by a thick arachnoid or deep sulcal location. Also, preanastomosis ICG-VA identified a considerable number of unsuitable recipients with pathological flow patterns that were missed under white light.

Although it remains a clear limitation, recipient selection based on white-light visualization remains a daily practice even in specialized neurovascular centers. The most common strategy is to select the recipient with the largest diameter, which facilitates suturing especially in moyamoya patients with a small vessel size and extremely fragile vessel wall. Moreover, a large diameter is hypoth- esized to correlate with high outflow as a primary determinant of bypass function ${ }^{1}$ and indicator for greater hemodynamic need. ${ }^{26-28}$ In our series, however, there was no association between recipient diameter and recipient flow, and selection of the recipient with the largest diameter appeared to have no significant influence on bypass function. Naturally, this could be at least partially due to the FLOW 800-specific blood flow measurement, which is an indicator dilution approach and subject to inhomogeneous mixing of the dye, varying bolus injection speed, blood volume, cardiac output, and individual angioarchitecture. On the other hand, our findings suggest that recipient selection in patients with hemodynamic compromise should be based on more information than recipient diameter alone.

Missing hemodynamic need with high vascular resistance is regarded as one of the main reasons for bypass failure. In our study, bypass function was expressed as the recipient flow increase, which ranged from near $0 \%$ to $450 \%$. Further, recipients with an initially low flow, late appearance on ICG-VA, and retrograde flow direction demonstrated a significantly greater recipient flow increase. We believe that these characteristics indicate greater hemodynamic need with little vascular resistance because low flow and late appearance are likely caused by a severe proximal perfusion deficit. Similarly, an initially retrograde blood flow direction within potential recipients could indicate hemodynamic steal from these vascular territories. Importantly, both situations are arguably associated with low vascular resistance, which supports the hypothesis that the distribution dynamics of the ICG dye across the anastomosis and into the recipient bed serve as an indicator of bypass function. ${ }^{13}$ In general, complementary orientation of the fish-mouthed graft to promote filling of the recipient in the same direction as the initial flow direction is assumed to be beneficial for bypass function. In our series, however, orientation of the bypass graft had no notable influence on bypass function. Naturally, combining both heel and toe orientations of the donor 

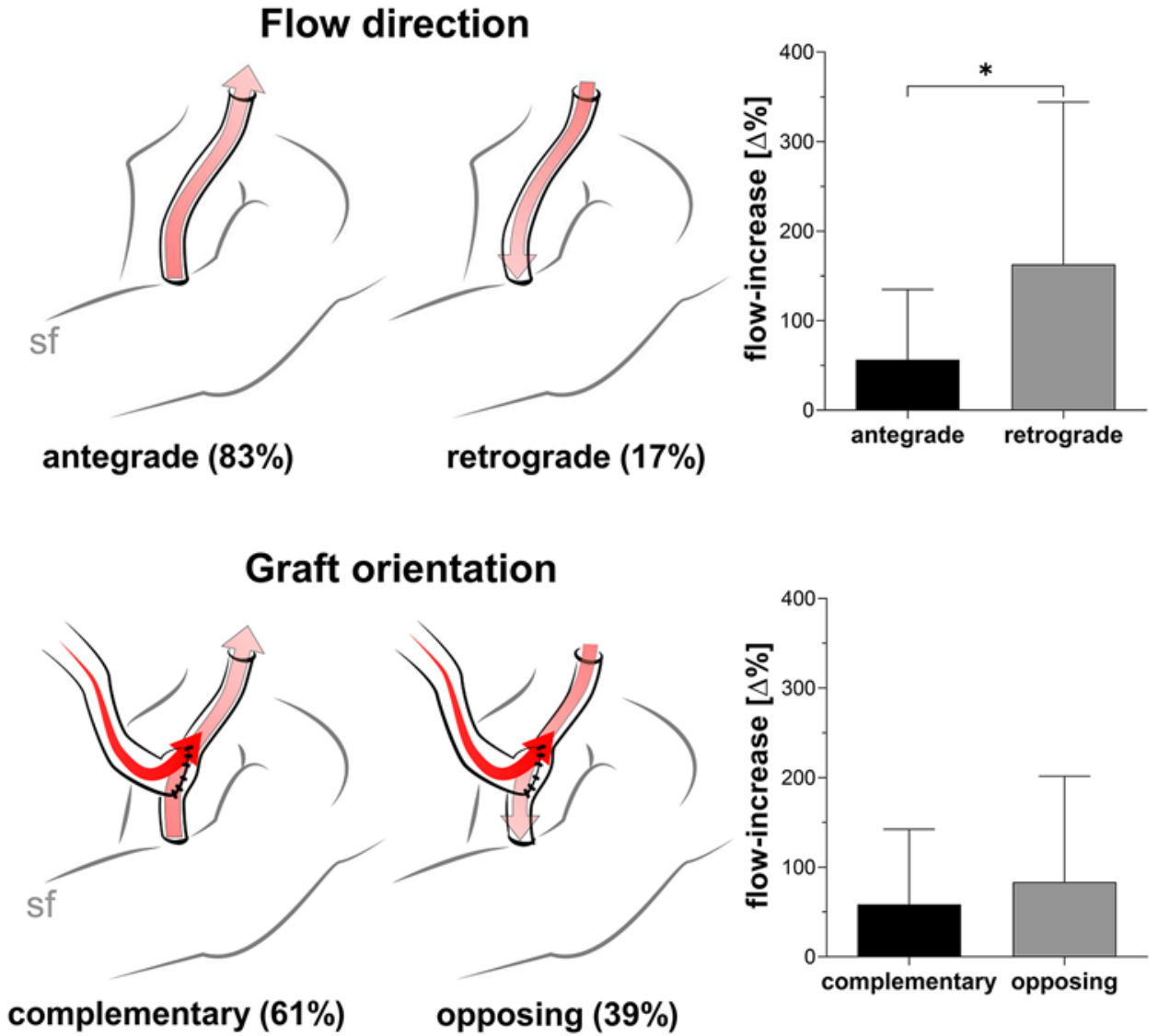

FIG. 6. Illustrations and bar graphs showing the influence of preanastomosis flow direction within the recipient (upper) and orientation of the fish-mouthed bypass graft (lower) on the recipient flow increase. Preanastomosis flow direction within the recipient was categorized as antegrade or retrograde in relation to the sylvian fissure (sf). Graft orientation was categorized as complementary or opposing depending on the orientation of the heel and toe of the donor to the preanastomosis blood flow direction within the recipient. Only retrograde flow direction was associated with a significant increase of the relative recipient flow $\left({ }^{*} p=0.02\right)$. Figure is available in color online only.

with both possible recipient blood flow directions results in four possible combinations. However, outflow of the donor overruled the inherent flow within the recipient in $96 \%$ of cases, regardless of heel and toe orientation. This suggests that graft orientation is not a relevant modifier of STA-MCA bypass function in hemodynamic compromise, since outflow of the donor appears to overrule the inherent flow of the recipient. Considering all aspects around flow increase, graft orientation, and initial blood flow direction, we conclude that initial retrograde recipient flow is a sign of increased hemodynamic need with low vascular resistance, which may permit a higher flow increase. Since the donor overruled flow in the recipient in nearly all cases, we believe that it is not a question of how the heel and toe of the donor are oriented, but rather how much flow can be fed across the anastomosis depending on the vascular resistance of the recipient. Thus, heel and toe orientation likely only plays a role in the context of the recipient flow direction.

For assessment of absolute blood flow, quantitative Doppler measurement of the recipient would be considered the gold standard..$^{29}$ Unfortunately, this was not technically feasible because of the limited exposure of the recipient bed and the small recipient diameter. Alternatively,
Charbel and colleagues established the "cut flow index" as an intraoperative measure to characterize bypass function in a quantitative fashion. ${ }^{1}$ This index describes the ratio of the postanastomosis blood flow within the donor in relation to the free-flowing (preanastomosis) donor blood flow after it has been cut at the tip. Accordingly, a cut flow index of 1 would indicate that the full potential flow of the donor is fed through the anastomosis into the recipient. ${ }^{1,3}$ Importantly, the cut flow index takes the relevance of the recipient and its downstream resistance into account, and the fact that patients with intact cerebrovascular reactivity were previously shown to have lower cut flow indices (poorer bypass function) than patients with impaired cerebrovascular reactivity ${ }^{1}$ is in line with our hypothesis that recipient characteristics with low vascular resistance appear to benefit bypass function.

\section{Study Limitations}

Although our study inherently lacks power because of its retrospective nature and single-center design, the study cohort is representative of patients with cerebral hemodynamic impairment undergoing standardized STAMCA revascularization. Nevertheless, generalizability of our findings may be limited because of our study design 
and center-specific standard operating procedures. Also, quantitative Doppler measurements were not available and semiquantitative assessment of fluorescence intensity/rise time $(\mathrm{AI} / \mathrm{sec})$ with FLOW 800 remains a relative measure of flow. Correlating flow measurements and recipient properties with angiographic follow-up, clinical outcomes, and long-term bypass function remains the most important task to determine bypass effectiveness. Unfortunately, however, correlation of examined parameters to long-term angiographic findings was not feasible in the present retrospective setting because filling of the MCA territory is naturally influenced by spontaneous dural collateralization or collateral outgrowth through an ipsilateral encephalomyosynangiosis in patients with MMV, and a consistent, superselective injection of the frontal or parietal STA branch (depending on which was chosen for the anastomosis) was not always available. Thus, selective assessment of the effect of recipient properties on the long-term revascularization result did not seem justified. In the future, a prospective study of the influence of recipient selection according to different recipient vessel properties may help to overcome this limitation.

\section{Conclusions}

In this study, ICG-VA helped to reliably identify a variety of physiological and pathological recipient flow patterns, ICG-VA appearance sequences, recipient diameters, and blood flow directions in an array of recipients, which indicates the diversity of potentially available recipients and highlights the importance of an appropriate selection. Most importantly, a stratified recipient selection based on parameters that are related to bypass function could help improve long-term patient outcomes because limited intraoperative graft flow or even an occluded bypass will not immediately become clinically apparent in patients who undergo flow augmentation surgery for chronic cerebral hemodynamic impairment.

\section{Acknowledgments}

We thank Lucius Fekonja for the illustration in Fig. 6.

\section{References}

1. Amin-Hanjani S, Du X, Mlinarevich N, et al. The cut flow index: an intraoperative predictor of the success of extracranial-intracranial bypass for occlusive cerebrovascular disease. Neurosurgery. 2005;56(1)(suppl):75-85.

2. Derdeyn CP, Grubb RL Jr, Powers WJ. Cerebral hemodynamic impairment: methods of measurement and association with stroke risk. Neurology. 1999;53(2):251-259.

3. Stapleton CJ, Atwal GS, Hussein AE, et al. The cut flow index revisited: utility of intraoperative blood flow measurements in extracranial-intracranial bypass surgery for ischemic cerebrovascular disease. J Neurosurg. Published online September 6, 2019. doi:10.3171/2019.5.JNS19641

4. Zammar SG, El Tecle NE, El Ahmadieh TY, et al. Impact of a vascular neurosurgery simulation-based course on cognitive knowledge and technical skills in European neurosurgical trainees. World Neurosurg. 2015;84(2):197-201.

5. Rychen J, Goldberg J, Raabe A, Bervini D. Augmented reality in superficial temporal artery to middle cerebral artery bypass surgery: technical note. Oper Neurosurg (Hagerstown). 2020;18(4):444-450.
6. Cabrilo I, Schaller K, Bijlenga P. Augmented reality-assisted bypass surgery: embracing minimal invasiveness. World Neurosurg. 2015;83(4):596-602.

7. Kikuta K, Takagi Y, Fushimi Y, et al. "Target bypass": a method for preoperative targeting of a recipient artery in superficial temporal artery-to-middle cerebral artery anastomoses. Neurosurgery. 2006;59(4)(suppl 2):ONS320-ONS327.

8. Raabe A, Nakaji P, Beck J, et al. Prospective evaluation of surgical microscope-integrated intraoperative near-infrared indocyanine green videoangiography during aneurysm surgery. J Neurosurg. 2005;103(6):982-989.

9. Kamp MA, Slotty P, Turowski B, et al. Microscope-integrated quantitative analysis of intraoperative indocyanine green fluorescence angiography for blood flow assessment: first experience in 30 patients. Neurosurgery. 2012;70(1)(Suppl Operative):65-74.

10. Nakagawa S, Murai Y, Matano F, et al. Evaluation of patency after vascular anastomosis using quantitative evaluation of visualization time in indocyanine green video angiography. World Neurosurg. 2018;110:e699-e709.

11. Yokota H, Yonezawa T, Yamada T, et al. Transdural indocyanine green videography for superficial temporal artery-tomiddle cerebral artery bypass-technical note. World Neurosurg. 2017;106:446-449.

12. Prinz V, Hecht N, Kato N, Vajkoczy P. FLOW 800 allows visualization of hemodynamic changes after extracranial-tointracranial bypass surgery but not assessment of quantitative perfusion or flow. Neurosurgery. 2014;10(suppl 2):231-239.

13. Januszewski J, Beecher JS, Chalif DJ, Dehdashti AR. Flowbased evaluation of cerebral revascularization using nearinfrared indocyanine green videoangiography. Neurosurg Focus. 2014;36(2):E14.

14. Awano T, Sakatani K, Yokose N, et al. Intraoperative EC-IC bypass blood flow assessment with indocyanine green angiography in moyamoya and non-moyamoya ischemic stroke. World Neurosurg. 2010;73(6):668-674.

15. Woitzik J, Horn P, Vajkoczy P, Schmiedek P. Intraoperative control of extracranial-intracranial bypass patency by nearinfrared indocyanine green videoangiography. J Neurosurg. 2005;102(4):692-698.

16. Czabanka M, Peña-Tapia P, Scharf J, et al. Characterization of direct and indirect cerebral revascularization for the treatment of European patients with moyamoya disease. Cerebrovasc Dis. 2011;32(4):361-369.

17. Wessels L, Hecht N, Vajkoczy P. Bypass in neurosurgeryindications and techniques. Neurosurg Rev. 2019;42(2): 389-393.

18. Vajkoczy P, Horn P, Schmiedek P. Standard superficial temporal artery-middle cerebral artery bypass surgery in hemodynamic cerebral ischemia: indication and technique. Oper Tech Neurosurg. 1999;2(3):106-115.

19. Acker G, Schlinkmann N, Fekonja L, et al. Wound healing complications after revascularization for moyamoya vasculopathy with reference to different skin incisions. Neurosurg Focus. 2019;46(2):E12.

20. Peña-Tapia PG, Kemmling A, Czabanka M, et al. Identification of the optimal cortical target point for extracranial-intracranial bypass surgery in patients with hemodynamic cerebrovascular insufficiency. J Neurosurg. 2008;108(4):655-661.

21. Kuebler WM, Sckell A, Habler O, et al. Noninvasive measurement of regional cerebral blood flow by near-infrared spectroscopy and indocyanine green. J Cereb Blood Flow Metab. 1998;18(4):445-456.

22. Esposito G, Dias S, Burkhardt JK, et al. Role of indocyanine green videoangiography in identification of donor and recipient arteries in cerebral bypass surgery. Acta Neurochir Suppl. 2018;129:85-89.

23. Esposito G, Durand A, Van Doormaal T, Regli L. Selectivetargeted extra-intracranial bypass surgery in complex middle 
cerebral artery aneurysms: correctly identifying the recipient artery using indocyanine green videoangiography. Neurosurgery. 2012;71(2)(Suppl Operative):ons274-ons285.

24. Esposito G, Regli L. Selective targeted cerebral revascularization via microscope integrated indocyanine green videoangiography technology. Acta Neurochir Suppl. 2014;119: 59-64.

25. Rodríguez-Hernández A, Lawton MT. Flash fluorescence with indocyanine green videoangiography to identify the recipient artery for bypass with distal middle cerebral artery aneurysms: operative technique. Neurosurgery. 2012;70(2) (Suppl Operative):209-220.

26. Yonas H, Pindzola RR. Effect of acetazolamide reactivity and long-term outcome in patients with major cerebral artery occlusive diseases. Stroke. 1998;29(8):1742-1744.

27. Schmiedek P, Piepgras A, Leinsinger G, et al. Improvement of cerebrovascular reserve capacity by EC-IC arterial bypass surgery in patients with ICA occlusion and hemodynamic cerebral ischemia. J Neurosurg. 1994;81(2):236-244.

28. Powers WJ, Clarke WR, Grubb RL Jr, et al. Extracranialintracranial bypass surgery for stroke prevention in hemodynamic cerebral ischemia: the Carotid Occlusion Surgery Study randomized trial. JAMA. 2011;306(18):1983-1992. Published correction in JAMA. 2011;306(24):2672.

29. Charbel FT, Hoffman WE, Misra M, Ostergren L. Ultrasonic perivascular flow probe: technique and application in neurosurgery. Neurol Res. 1998;20(5):439-442.

\section{Disclosures}

The authors report no conflict of interest concerning the materials or methods used in this study or the findings specified in this paper.

\section{Author Contributions}

Conception and design: Hecht. Acquisition of data: Hecht, Goldberg. Analysis and interpretation of data: all authors. Drafting the article: Hecht, Goldberg. Critically revising the article: all authors. Reviewed submitted version of manuscript: all authors. Approved the final version of the manuscript on behalf of all authors: Hecht. Statistical analysis: Hecht, Goldberg. Administrative/technical/material support: Vajkoczy. Study supervision: Hecht, Vajkoczy.

\section{Supplemental Information Previous Presentations}

Portions of this work were presented orally at the EANS Vascular Section Meeting held in Nice, France, on September 5-6, 2019.

\section{Correspondence}

Peter Vajkoczy: Charité Universitätsmedizin Berlin, Germany. peter.vajkoczy@charite.de. 